PROCEEDINGS OF THE

AMERICAN MATHEMATICAL SOCIETY

Volume 136, Number 9, September 2008, Pages 3133-3143

S 0002-9939(08)09309-X

Article electronically published on May 5, 2008

\title{
A SCHWARZ LEMMA FOR MEROMORPHIC FUNCTIONS AND ESTIMATES FOR THE HYPERBOLIC METRIC
}

\author{
ALEXANDER YU. SOLYNIN
}

(Communicated by Ronald A. Fintushel)

\begin{abstract}
We prove a generalization of the Schwarz lemma for meromorphic functions $f$ mapping the unit disk $\mathbb{D}$ onto Riemann surfaces $\mathcal{R}$ with bounded in mean radial distances from $f(0)$ to the boundary of $\mathcal{R}$. A new variant of the Schwarz lemma is also proved for the Carathèodory class of analytic functions having positive real part in $\mathbb{D}$. Our results lead to several improved estimates for the hyperbolic metric.
\end{abstract}

\section{INTRODUCTION AND RESULTS}

Let $f$ be analytic on the unit disk $\mathbb{D}=\{z:|z|<1\}$. By the classical Schwarz lemma, the inequalities

$$
\text { (a) }\left|f^{\prime}(0)\right| \leq 1 \text { and (b) }|f(z)-f(0)| \leq|z| \text { for every } z \in \mathbb{D}
$$

hold for all $f$ such that

$$
\sup _{z \in \mathbb{D}}|f(z)-f(0)| \leq 1 .
$$

In Theorem 1 below, we show that (1.1) (a) remains valid when (1.2) is replaced by a much weaker condition that the geometric mean of the distance from $f(0)$ to the boundary of $f(\mathbb{D})$ is bounded by 1 . Theorem 4 gives a similar improvement for the variant of Schwarz lemma for analytic functions with positive real part. Our results lead to several new estimates for the hyperbolic metric of planar domains.

Let $f$ be meromorphic on $\mathbb{D}$ having an expansion

$$
f(z)=a_{p} z^{p}+a_{p+1} z^{p+1}+\cdots
$$

at $z=0$. Here $p$ is an integer $\geq 1$ and $a_{p} \neq 0$. Let $\mathcal{R}_{f}$ be the Riemann surface of $f^{-1}$ and let $P_{0}$ be the point on $\mathcal{R}_{f}$ such that $f^{-1}\left(P_{0}\right)=0$. We note that $P_{0}$ is a branch point of $\mathcal{R}_{f}$ of order $p-1$ if $p \geq 2$. For a fixed $\delta>0$ sufficiently small, the vector $\vec{v}(\varphi)=\delta e^{i \varphi}$, where $0 \leq \varphi<2 \pi p$, can be thought of as a direction vector on $\mathcal{R}_{f}$. Let $\mathcal{B}$ be the set (at most countable) of all branch points $P \neq P_{0}$ of $\mathcal{R}_{f}$.

Let $d_{P_{0}}(\varphi, f(\mathbb{D}))$ denote the Euclidean distance from $P_{0}$ to $\partial \mathcal{R}_{f} \cup \mathcal{B}$ measured on $\mathcal{R}_{f}$ in the direction defined by $\vec{v}(\varphi)$. We emphasize that for $1 \leq k<p$, the

Received by the editors April 30, 2007.

2000 Mathematics Subject Classification. Primary 30C80.

Key words and phrases. Schwarz lemma, meromorphic function, hyperbolic metric, reduced module, polarization.

This research was supported in part by NSF grant DMS-0525339.

(C)2008 American Mathematical Society 
distances $d_{P_{0}}(\varphi, f(\mathbb{D}))$ and $d_{P_{0}}(\varphi+2 \pi k, f(\mathbb{D}))$ are measured on different sheets of $\mathcal{R}_{f}$ and therefore $d_{P_{0}}(\varphi, f(\mathbb{D})) \neq d_{P_{0}}(\varphi+2 \pi k, f(\mathbb{D}))$ for such $k$, in general.

Theorem 1. Let $f$ be meromorphic on $\mathbb{D}$ having expansion (1.3) at $z=0$. Then

$$
\left|a_{p}\right| \leq \exp \left\{\frac{1}{2 \pi p} \int_{0}^{2 \pi p} \log d_{P_{0}}(t, f(\mathbb{D})) d t\right\} .
$$

Further, equality holds in (1.4) if and only if $f(z)=c z^{p}$ for some $c \in \mathbb{C} \backslash\{0\}$.

The proof of Theorem 1 is given in Section 3 . Section 2 contains necessary results on the reduced modules of simply connected domains and triangles.

In 1907, Landau and Toeplitz proved that (1.1) (a) remains valid when (1.2) is replaced by the condition Diam $f(\mathbb{D}) \leq 2$. This result, along with many others, was discussed recently in [2]. Theorem 1 implies the following stronger form of the Landau-Toeplitz theorem.

Theorem 2. Under the assumptions of Theorem 1 suppose, in addition, that the length of every straight line segment embedded into $\mathcal{R}_{f}$, which passes through $P_{0}$, does not exceed 2 . Then $\left|a_{p}\right| \leq 1$ with the sign of equality if and only if $f(z)=c z^{p}$ for some $c$ such that $|c|=1$.

In particular, if $\operatorname{Diam} f(\mathbb{D}) \leq 2$, then the previous conclusion holds.

Proof. By the arithmetic-geometric mean inequality and our assumption,

$$
\sqrt{d_{P_{0}}(t, f(\mathbb{D})) d_{P_{0}}(t+\pi p, f(\mathbb{D}))} \leq\left(d_{P_{0}}(t, f(\mathbb{D}))+d_{P_{0}}(t+\pi p, f(\mathbb{D}))\right) / 2 \leq 1
$$

for every $t, 0 \leq t \leq \pi p$. This together with (1.4) implies that

$$
\left|a_{p}\right| \leq \exp \left\{\frac{1}{2 \pi p} \int_{0}^{\pi p} \log \left(d_{P_{0}}(t, f(\mathbb{D})) d_{P_{0}}(t+\pi p, f(\mathbb{D}))\right) d t\right\} \leq 1 .
$$

By Theorem 1, equality holds in the first inequality in (1.5) if and only if $f(z)=c z^{p}$ for some $c \in \mathbb{C}$. Then equality occurs in the second of these inequalities if and only if $|c|=1$.

In 1938, L. Ahlfors (see 1]) extended the Schwarz lemma by considering analytic maps from the disk to a Riemann surface. Ahlfors' result shows, in particular, that analytic maps are contractions in the hyperbolic metric. For planar domains, the hyperbolic metric can be defined as follows.

Let $\Omega$ be a domain on $\overline{\mathbb{C}}$. If the complement $\overline{\mathbb{C}} \backslash \Omega$ contains at least three points, then $\Omega$ has the unit disk $\mathbb{D}$ as its universal covering surface. If $\Pi: \mathbb{D} \rightarrow \Omega$ is a universal covering map, the hyperbolic (or Poincaré) density $\lambda_{\Omega}$ for $\Omega$ is then given by

$$
\lambda_{\Omega}(\Pi(z))\left|\Pi^{\prime}(z)\right|=1 /\left(1-|z|^{2}\right), \quad \text { where } z \in \mathbb{D}, \quad w=\Pi(z) .
$$

For further properties and applications of the hyperbolic metric we refer to [1] and [4, Ch. 9].

Theorem 3. Let $\Omega$ be a domain in $\overline{\mathbb{C}}$ endowed with the hyperbolic metric $\lambda_{\Omega}$. If $z_{0} \in \Omega$ is finite, then

$$
\lambda_{\Omega}\left(z_{0}\right) \geq \exp \left\{-\frac{1}{2 \pi} \int_{0}^{2 \pi} \log d_{z_{0}}(t, \Omega) d t\right\}
$$

with the sign of equality if and only if $\Omega$ is a disk centered at $z_{0}$. 
In particular, if $\frac{1}{2 \pi} \int_{0}^{2 \pi} \log d_{z}(t, \Omega) d t \leq 0$ for all finite $z \in \Omega$, then

$$
\inf _{z \in \Omega} \lambda_{\Omega}(z) \geq 1 \text {. }
$$

If, in addition, $\Omega$ is bounded, then $\lambda_{\Omega}(z)$ achieves its minimal value $m \geq 1$ at some point $z_{0} \in \Omega$. If $m=1$, then $\Omega$ is a disk of radius 1 centered at $z_{0}$.

Proof. Let $\Pi: \mathbb{D} \rightarrow \Omega$ be a universal covering map such that $\Pi(0)=z_{0}$. By (1.6), $\lambda_{\Omega}\left(z_{0}\right)=\left|\Pi^{\prime}(0)\right|^{-1}$. Now (1.7) together with the assertion on the cases of equality follows from Theorem 1 applied to $\Pi(z)$.

If $\frac{1}{2 \pi} \int_{0}^{2 \pi} \log d_{z_{0}}(t, \Omega) d t \leq 0$ for all $z_{0} \in \Omega$, then (1.7) also holds for every $z_{0} \in \Omega$, which implies (1.8). If, in addition, $\Omega$ is bounded, then $\lambda_{\Omega}(z)$ is continuous in $\Omega$ and $\lambda_{\Omega}(z) \rightarrow \infty$ as $z \rightarrow \partial \Omega$; see [4, Ch. 9]. Therefore $\lambda_{\Omega}(z)$ achieves its minimum $m \geq 1$ at some point $z_{0} \in \Omega$; i.e. $\lambda_{\Omega}\left(z_{0}\right)=m$. Finally, if $m=1$, then, for such $z_{0}$, equation (1.7) holds with the sign of equality, which in its turn implies that $\Omega$ is a disk of radius 1 centered at $z_{0}$.

Corollary 1. If $\lambda_{\Omega}(z) \leq 1$ for at least one point $z \in \Omega$, then $\Omega$ either contains a closed segment of length 2 or it is a disk of radius 1 .

Proof. If $\Omega$ contains no closed segments of length $\geq 2$, then $d_{z}(t, \Omega)+d_{z}(t+\pi, \Omega) \leq$ 2 for all $z \in \Omega$ and every $t, 0 \leq t<2 \pi$. Applying (1.7) and the arithmeticgeometric mean inequality as in the proof of Theorem 2, we conclude that $\lambda_{\Omega}(z) \geq$ $\exp \left\{-\frac{1}{2 \pi} \int_{0}^{2 \pi} \log d_{z}(t, \Omega) d t\right\} \geq 1$ for every $z \in \Omega$. Moreover, if $\lambda_{\Omega}\left(z_{0}\right)=1$ for some $z_{0} \in \Omega$, then $\Omega$ must be the disk of radius 1 centered at $z_{0}$.

Next we consider the set $\mathcal{P}$ of all functions $f$ analytic in $\mathbb{D}$ such that $f(0)=1$ and $\Re f(z)>0$ for $z \in \mathbb{D}$. Thus, $\mathcal{P}$ is a well-known Carathéodory class of analytic functions having positive real part in $\mathbb{D}$. If $f \in \mathcal{P}$, then

$$
\text { (a) }\left|f^{\prime}(0)\right| \leq 2 \quad \text { and } \quad\left(\text { b) } \quad|f(z)-1| \leq \frac{2|z|}{1-|z|} \quad \text { for every } z \in \mathbb{D}\right. \text {. }
$$

The inequalities in (1.9) play the role of a Schwarz lemma for the class $\mathcal{P}$. Our next theorem, the proof of which is given in Section 5 generalizes this for a much broader class of functions.

Theorem 4. Let $f$ be analytic in $\mathbb{D}$ with $f(0)=1$ such that for every $v \in \mathbb{R}$ the set $\{w=t+i v: 0 \leq t \leq 2\} \backslash f(\mathbb{D})$ is not empty. Then inequalities (1.9) remain valid.

Further, equality holds in (1.9) (a) or in (1.9) (b) for some $z \in \mathbb{D} \backslash\{0\}$ if and only if $f(z)=f_{\alpha}(z)$ or $f(z)=2-f_{\alpha}(z)$ for some $\alpha \in \mathbb{R}$, where $f_{\alpha}(z)=\left(1-e^{i \alpha} z\right) /(1+$ $\left.e^{i \alpha} z\right)$.

Corollary 2. If $\Omega \cap \mathbb{R} \neq \emptyset$ and $\lambda_{\Omega}(x) \leq 1$ for at least one point $x \in \Omega \cap \mathbb{R}$, then $\Omega$ either contains a closed vertical segment of length 1 , which is symmetric with respect to the real axis, or it is one of the following two half-planes: $H^{+}=\{z: \Im z>-1 / 2\}$ or $H^{-}=\{z: \Im z<1 / 2\}$.

Proof. Suppose that $\lambda_{\Omega}\left(x_{0}\right) \leq 1$ for some $x_{0} \in \mathbb{R}$. Let $\Pi: \mathbb{D} \rightarrow \Omega$ be a universal covering map such that $\Pi(0)=x_{0}$ and let $f(z)=1+2 i\left(\Pi(z)-x_{0}\right)$. Then by (1.6),

$$
\left|f^{\prime}(0)\right|=2\left|\Pi^{\prime}(0)\right|=2 \lambda_{\Omega}^{-1}\left(x_{0}\right) \geq 2 .
$$

Since $f$ satisfies the assumptions of Theorem 4 , the strict inequality in (1.10) will contradict (1.9) (a). If the last relation of (1.10) holds with the sign of equality, 
then by Theorem 4, $f(z)=f_{\alpha}(z)$ or $f(z)=2-f_{\alpha}(z)$ for some $\alpha \in \mathbb{R}$. The latter shows that $\Omega$ must be one of the half-planes $H^{+}$or $H^{-}$.

\section{Reduced MOdUli OF CONFORMAL CONFIGURATIONS}

Let $D$ be a hyperbolic simply connected domain on a Riemann surface $\mathcal{R}$ and $P_{0} \in D$. Let us fix a chart $(U, \varphi)$ on $\mathcal{R}$ such that $\varphi$ maps $U$ onto the disk $\mathbb{D}_{\varepsilon_{0}}=$ $\left\{\zeta:|\zeta|<\varepsilon_{0}\right\}$ for some $\varepsilon_{0}>0$ and $\varphi\left(P_{0}\right)=0$. If $0<\varepsilon<\varepsilon_{0}$, then $D(\varepsilon)=D \backslash\{P \in$ $\mathcal{R}:|\varphi(P)|<\varepsilon\}$ is a doubly-connected domain on $\mathcal{R}$. Let $\bmod (D(\varepsilon))$ denote the modulus of $D(\varepsilon)$; i.e., $\bmod (D(\varepsilon))=(1 / 2 \pi) \log \left(r_{2} / r_{1}\right)$ if $D(\varepsilon)$ is conformally equivalent to an annulus $A\left(r_{1}, r_{2}\right)=\left\{z: r_{1}<|z|<r_{2}\right\}$.

The reduced modulus $m_{\zeta}\left(D, P_{0}\right)$ of $D$ at $P_{0}$ with respect to the uniformizing parameter $\zeta=\varphi(P)$ is then defined by

$$
m_{\zeta}\left(D, P_{0}\right)=\lim _{\varepsilon \rightarrow 0}(\bmod (D(\varepsilon))+(1 / 2 \pi) \log \varepsilon),
$$

where the finite limit exists; cf. [5, Theorem 2.8].

Let $T=T\left(v_{0}, v_{1}, v_{2}\right)$ be a triangle in $D$ having the vertex $v_{0}$ at $P_{0}$ and the side $e_{0}$ opposite to $v_{0}$ on $\partial D$. Here by a triangle $T=T\left(v_{0}, v_{1}, v_{2}\right)$ we mean a configuration consisting of a simply connected domain $T$ with three distinct boundary points $v_{0}, v_{1}, v_{2}$ (called vertices) marked on $\partial T$. Then the set $\partial T \backslash\left\{v_{0}, v_{1}, v_{2}\right\}$ consists of three sides, $e_{0}, e_{1}$, and $e_{2}$, where $e_{k}$ is the side opposite to $v_{k}$.

Suppose further that for all sufficiently small $\varepsilon>0$ the set $T \cap\{P:|\varphi(P)|=\varepsilon\}$ consists of a single arc $\gamma_{\varepsilon}$ and the boundary of $\varphi(T \cap U)$ forms an interior angle $\psi, 0<\psi \leq 2 \pi$, at $\zeta=0$. Then the set $T(\varepsilon)=T \backslash\{P:|\varphi(P)| \leq \varepsilon\}$ may be considered as a quadrilateral having $e_{0}$ and $\gamma_{\varepsilon}$ as a distinguished pair of its sides. Let $\bmod (T(\varepsilon))$ be the modulus of $T(\varepsilon)$ with respect to the family of curves separating $e_{0}$ from $\gamma_{\varepsilon}$ inside $T(\varepsilon)$; see [5, Section 2.3].

The reduced modulus $m_{\zeta}\left(T ; v_{0} \mid v_{1}, v_{2}\right)$ of the triangle $T$ at its vertex $v_{0}$ with respect to the uniformizing parameter $\zeta$ is defined by

$$
m_{\zeta}\left(T ; v_{0} \mid v_{1}, v_{2}\right)=\lim _{\varepsilon \rightarrow 0}(\bmod (T(\varepsilon))+(1 / \psi) \log \varepsilon),
$$

provided that the finite limit exists; see [10, §1.2].

The definition (2.1) of the reduced modulus of a simply connected domain is classical due to O. Teichmüller. The definition (2.2) of the reduced modulus of a triangle is reminiscent of Teichmüller's definition (2.1). It was introduced by this author for planar triangles in [8] and for triangles on Riemann surfaces in [10]. We want to emphasize here that the limit in (2.2) may not exist even if the sides of $T$ are analytic arcs; see [10, Example 1.1]. However, if for all sufficiently small $\varepsilon>0$, $(\partial \varphi(T \cap U)) \cap \mathbb{D}_{\varepsilon}$ consists of two straight line segments, an easy symmetry and reflection argument shows that the finite reduced modulus $m_{\zeta}\left(T ; v_{0} \mid v_{1}, v_{2}\right)$ exists.

Let $\mathcal{R}_{1}$ be a Riemann surface, $Q_{0} \in \mathcal{R}_{1}$, and let $\left(U_{1}, \varphi_{1}\right)$ be a chart on $\mathcal{R}_{1}$ such that $\varphi_{1}\left(Q_{0}\right)=0$. If $f$ maps $D$ conformally onto a domain $\Omega, Q_{0} \in \Omega \subset \mathcal{R}_{1}$, such that $f\left(P_{0}\right)=Q_{0}$ and $\tau(\zeta)=\varphi_{1}\left(f\left(\varphi^{-1}(\zeta)\right)\right)$, then it is well-known that

$$
m_{\tau}\left(\Omega, Q_{0}\right)=m_{\zeta}\left(D, P_{0}\right)+(1 / 2 \pi) \log \left|\tau^{\prime}(0)\right| .
$$

A similar formula holds for the change of the reduced modulus of a triangle:

$$
m_{\tau}\left(f(T) ; Q_{0} \mid Q_{1}, Q_{2}\right)=m_{\zeta}\left(T ; P_{0} \mid P_{1}, P_{2}\right)+\left(1 / \psi_{1}\right) \log \left|A_{1}\right| ;
$$


see [10, Lemma 1.3]. In (2.4), we assume that $f$ maps $T$ conformally onto the triangle $f(T)$ such that $f\left(P_{k}\right)=Q_{k}, k=0,1,2$, and that $\tau=\tau(\zeta)$ has the following expansion near $\zeta=0$ :

$$
\tau(\zeta)=A_{1} \zeta^{\psi_{1} / \psi}(1+g(\zeta))
$$

where $A_{1} \neq 0$ and $g$ is analytic at $\zeta=0$ such that $g(0)=0$.

Next we consider the partitioning of a simply connected domain $D$ or a triangle $T$ on $\mathcal{R}$ into $n$ nonoverlapping triangles $T_{1}, \ldots, T_{n}$. In case of partitioning of a domain, we assume that $T_{k} \subset D$ and every $T_{k}=T_{k}\left(v_{0, k}, v_{1, k}, v_{2, k}\right)$ has its vertex $v_{0, k}$ at $P_{0} \in D$ and the opposite side $e_{0, k}$ on $\partial D$. We assume further that $T_{k}$ has a nonzero angle $2 \pi \alpha_{k}$ at $v_{0, k}$ and the reduced modulus $m_{\zeta}\left(T_{k} ; v_{0, k} \mid v_{1, k}, v_{2, k}\right)$ exists for every $1 \leq k \leq n$.

In the case of the partitioning of a triangle $T=T\left(v_{0}, v_{1}, v_{2}\right)$, we assume that $T_{k} \subset T, T$ and $T_{k}$ have their vertices $v_{0}$ and $v_{0, k}$ at the same point $P_{0} \in \mathcal{R}$, and the side $e_{0, k}$ opposite to $v_{0, k}$ lies on the side $e_{0}$ of $T$. In addition, we assume that $T$ has the angle $0<\beta \leq 2 \pi$ at $v_{0}$ and $T_{k}$ has the angle $\beta \alpha_{k}$ at $v_{0, k}$, and the reduced moduli $m_{\zeta}\left(T ; v_{0} \mid v_{1}, v_{2}\right)$ and $m_{\zeta}\left(T_{k} ; v_{0, k} \mid v_{1, k}, v_{2, k}\right)$ exist for all $1 \leq k \leq n$. In all cases under consideration we assume that $0<\alpha_{k} \leq 1$ for $1 \leq k \leq n$ and $\sum_{k=1}^{n} \alpha_{k}=1$.

Let $f_{D}$ map $D$ conformally onto $\mathbb{D}$ such that $f_{0}\left(P_{0}\right)=0$ and let $f_{T}$ map $T$ conformally onto the circular sector $S_{\beta}(1)$ such that $f_{T}\left(v_{0}\right)=0, f_{T}\left(v_{1}\right)=1$, and $f_{T}\left(v_{2}\right)=e^{i \beta}$. Here $S_{\beta}(R)=\{z: 0<|z|<R, 0<\arg z<\beta\}, 0<R \leq \infty$.

Lemma 1 ([8, [10]). (1) Let $T_{1}, \ldots, T_{n}$ be a partitioning of $D$ defined above. Then

$$
m_{\zeta}\left(D, P_{0}\right) \leq \sum_{k=1}^{n} \alpha_{k}^{2} m_{\zeta}\left(T_{k} ; v_{0, k} \mid v_{1, k}, v_{2, k}\right)
$$

Further, equality holds in (2.5) if and only if for every $k, 1 \leq k \leq n, f_{D}\left(T_{k}\right)$ is a circular sector of opening $2 \pi \alpha_{k}$ and $f_{D}\left(e_{0, k}\right)$ is a circular part of $\partial f_{D}\left(T_{k}\right)$.

(2) Let $T_{1}, \ldots, T_{n}$ be a partitioning of a triangle $T$ defined above. Then

$$
m_{\zeta}\left(T ; v_{0} \mid v_{1}, v_{2}\right) \leq \sum_{k=1}^{n} \alpha_{k}^{2} m_{\zeta}\left(T_{k} ; v_{0, k} \mid v_{1, k}, v_{2, k}\right) .
$$

Further, equality holds in (2.6) if and only if for every $k, 1 \leq k \leq n, f_{T}\left(T_{k}\right)$ is a circular sector of opening $\beta \alpha_{k}$ and $f_{T}\left(e_{0, k}\right)$ is a circular part of $\partial f_{T}\left(T_{k}\right)$.

For the proof of (2.5) and (2.6), which uses only basic facts of the theory of extremal metrics, we refer to [10, §1].

Now we consider a planar triangle $T=T\left(v_{0}, v_{1}, v_{2}\right)$ in the $z$-plane lying in the wedge $S_{\alpha}(\infty), 0<\alpha \leq 2 \pi$. Let $T$ have its vertices at the points $v_{0}=0, v_{1}=r_{1}>0$, and $v_{2}=r_{2} e^{i \alpha}, r_{2}>0$, and let $e_{1}=\left\{t e^{i \alpha}: 0<t<r_{2}\right\}$ and $e_{2}=\left\{t: 0<t<r_{1}\right\}$ be the sides of $T$ issuing from $v_{0}$. Suppose further that $T$ is starlike with respect to its vertex $v_{0}$. The latter means that the open interval $(0, z)$ joining 0 and $z$ is in $T$ whenever $z \in T$.

Lemma 2. Suppose that $T=T\left(v_{0}, v_{1}, v_{2}\right)$ is a starlike triangle defined above. Then

$$
m_{z}\left(T ; v_{0} \mid v_{1}, v_{2}\right) \leq \alpha^{-2} \int_{0}^{\alpha} \log d_{0}(t, T) d t .
$$

Equality holds in (2.7) if and only if $T=S_{\alpha}(R)$ for some $0<R<\infty$ and $v_{0}, v_{1}$, $v_{2}$ are the geometric vertices of $S_{\alpha}(R)$. 
Proof. We may assume that $L_{0}=\int_{0}^{\alpha} \log d_{0}(t, T) d t<\infty$. First, we approximate $T$ by a sequence of triangles $T^{(n)}=T^{(n)}\left(v_{0}^{n}, v_{1}^{n}, v_{2}^{n}\right)$ such that the side $e_{0}^{n}$ of $T^{(n)}$ opposite to $v_{0}^{n}=0$ consists of $n$ circular arcs and $n-1$ radial segments (possibly degenerate) joining these arcs. The precise construction is as follows.

For a fixed $n \geq 1$ and $1 \leq k \leq n$, let $d_{n, k}=\min d_{0}(t, T)$, where the minimum is taken over all $t$ such that $\alpha(k-1) / n \leq t \leq \alpha k / n$. The circular sectors

$$
T^{n, k}=\left\{z:|z|<d_{n, k}, \alpha(k-1) / n<\arg z<\alpha k / n\right\}, \quad 1 \leq k \leq n,
$$

will be considered as triangles $T^{n, k}\left(v_{0}^{n, k}, v_{1}^{n, k}, v_{2}^{n, k}\right)$ with vertices

$$
v_{0}^{n, k}=0, \quad v_{1}^{n, k}=d_{n, k} e^{i \alpha(k-1) / n}, \quad v_{2}^{n, k}=d_{n, k} e^{i \alpha k / n} .
$$

Now let $T^{(n)}$ be the interior of the closure of the union $\bigcup_{k=1}^{n} T^{n, k}$. Then $T^{(n)}$ can be considered as a triangle with vertices $v_{0}^{n}=0, v_{1}^{n}=d_{n, k}$, and $v_{2}^{n}=d_{n, k} e^{i \alpha}$. Since $d_{0}\left(t, T^{(n)}\right) \leq d_{0}\left(t, T^{(n+1)}\right) \leq d_{0}(t, T)$ for all $0 \leq t \leq \alpha$ and all $n \geq 1$, it follows from the Levi's theorem that

$$
\frac{\alpha}{n} \sum_{k=1}^{n} \log d_{n, k}=\int_{0}^{\alpha} \log d_{0}\left(t, T^{(n)}\right) d t \rightarrow \int_{0}^{\alpha} \log d_{0}(t, T) d t
$$

monotonically as $n \rightarrow \infty$.

Since $T^{(n)} \subset T^{(n+1)}$ and $\bigcup_{n=1}^{\infty} T^{(n)}=T$, we conclude that the sequence of domains $T^{(n)}$ converges to the kernel $T$. Then, using the Schwarz reflection principle and Carathéodory's theorem on the convergence to the kernel, we obtain that

$$
m_{z}\left(T^{(n)} ; v_{0}^{n} \mid v_{1}^{n}, v_{2}^{n}\right) \rightarrow m_{z}\left(T ; v_{0} \mid v_{1}, v_{2}\right) \quad \text { as } n \rightarrow \infty .
$$

Applying Lemma 1(2) with $\alpha_{k}=1 / n$ to the partitioning of $T^{(n)}$ into the triangles $T^{n, k}, k=1, \ldots, n$, we obtain the inequality

$$
m_{z}\left(T^{(n)} ; v_{0}^{n} \mid v_{1}^{n}, v_{2}^{n}\right) \leq \frac{1}{n^{2}} \sum_{k=1}^{n} m_{z}\left(T^{n, k} ; v_{0}^{n, k} \mid v_{1}^{n, k}, v_{2}^{n, k}\right)=\frac{1}{\alpha n} \sum_{k=1}^{n} \log d_{n, k},
$$

which, when combined with (2.8) and (2.9), implies (2.7).

Suppose now that (2.7) holds with the sign of equality. Let $r(\theta)=\{z: \arg z=$ $\theta$ \}. For $0<\theta<\alpha$, the ray $r(\theta)$ divides $T$ into two triangles, say $T_{1}$ and $T_{2}$. Here $T_{1}$ has vertices $v_{0}, v_{1}$, and $v^{\prime}=d_{0}(\theta, T) e^{i \theta}$ and $T_{2}$ has vertices $v_{0}, v^{\prime}$, and $v_{2}$. Let $\beta_{1}=\theta / \alpha, \beta_{2}=(\alpha-\theta) / \alpha$. By Lemma 1(2), we have

$$
m_{z}\left(T ; v_{0} \mid v_{1}, v_{2}\right) \leq \beta_{1}^{2} m_{z}\left(T_{1} ; v_{0} \mid v_{1}, v^{\prime}\right)+\beta_{2}^{2} m_{z}\left(T_{2} ; v_{0} \mid v^{\prime}, v_{2}\right) .
$$

Moreover, equality holds in (2.10) if and only if $f_{T}$ maps the interval $\left(0, d_{0}(\theta, T) e^{i \theta}\right)$ onto the radius $\left(0, e^{i \theta}\right)$. Here $f_{T}$ is a conformal mapping from $T$ onto the sector $S_{\alpha}(1)$ such that $f_{0}\left(v_{0}\right)=0, f_{0}\left(v_{1}\right)=1$, and $f_{0}\left(v_{2}\right)=e^{i \alpha}$.

Applying (2.7) to each of the triangles $T_{1}$ and $T_{2}$ separately and then combining the results, we obtain

$$
\beta_{1}^{2} m_{z}\left(T_{1} ; v_{0} \mid v_{1}, v^{\prime}\right)+\beta_{2}^{2} m_{z}\left(T_{2} ; v_{0} \mid v^{\prime}, v_{2}\right) \leq \alpha^{-2} \int_{0}^{\alpha} \log d_{0}(t, T) d t .
$$

It follows from (2.10) and (2.11) that if (2.7) holds with the sign of equality, we must have equality in (2.10) for every $\theta, 0<\theta<\alpha$. Then the equality assertion of Lemma 1(2) implies that $f_{T}$ maps every radial interval $\left(0, d_{0}(\theta, T) e^{i \theta}\right)$ onto the radius $\left(0, e^{i \theta}\right)$ of the sector $S_{\alpha}(1)$. Then $\arg (f(z) / z)=0$ for all $z \in T$. As is well 
known, the latter implies that $f(z) / z$ is constant on $T$. Hence, $f(z)=c z$ for some nonzero $c \in \mathbb{C}$. Therefore, $T$ must be a circular sector of opening $\alpha$.

Lemma 3. (1) Let $\mathcal{R}$ be a Riemann surface over the z-sphere $\overline{\mathbb{C}}, D$ a simply connected domain on $\mathcal{R}$, and $P_{0}$ a point of $D$ over $z=0$. Assume further that $P_{0}$ is a branch point of $\mathcal{R}$ of order $p-1 \geq 0$. In particular, if $p=1$, then $P_{0}$ is a regular point of $\mathcal{R}$. Let $\zeta=z^{1 / p}$ be a uniformizing parameter near $P_{0}$. Then

$$
m_{\zeta}\left(D, P_{0}\right) \leq 1 /(2 \pi p) \int_{0}^{2 \pi p} \log d_{P_{0}}(t, D) d t .
$$

Equality holds in (2.12) if and only if $D$ is a p-sheeted disk on $\mathcal{R}$ centered at $P_{0}$.

(2) Let $\mathcal{R}$ be a Riemann surface over $\overline{\mathbb{C}}, v_{0}$ a point of $\mathcal{R}$ over $z=0$, and $T=T\left(v_{0}, v_{1}, v_{2}\right)$ a triangle on $\mathcal{R}$ such that its sides $e_{1}$ and $e_{2}$ are straight line intervals on $\mathcal{R}$ forming an angle $\alpha>0$ at the vertex $v_{0}$. Let $v_{0}$ be a branch point of $\mathcal{R}$ of order $p-1 \geq 0$ and $\zeta=z^{1 / p}$ a uniformizing parameter near $v_{0}$. Then $0<\alpha \leq 2 \pi p$ and

$$
m_{\zeta}\left(T ; v_{0} \mid v_{1}, v_{2}\right) \leq \alpha^{-2} \int_{0}^{\alpha} \log d_{v_{0}}(t, T) d t .
$$

Equality holds in (2.13) if and only if $T$ is a circular sector on $\mathcal{R}$ of opening $\alpha$.

Proof. (a) Let $g_{D}$ be a conformal map from $\mathbb{D}$ onto $D$ such that $g_{D}(0)=P_{0}$. Let $r_{n} \rightarrow 1$ as $n \rightarrow \infty$ be an increasing sequence such that $g_{D}$ has no critical points on the circle $C_{r_{n}}$, where $C_{r}=\{z:|z|=r\}$. Let $D_{n}=g_{D}\left(\mathbb{D}_{r_{n}}\right)$ and $\gamma_{n}=g_{D}\left(C_{r_{n}}\right)$. Let $E_{n}=\left\{z_{P}: P \in \hat{E}_{n}\right\}$, where $z_{P}$ is the affix of $P \in \mathcal{R}$ and $\hat{E}_{n}$ is the set consisting of all branch points of $\mathcal{R}$ lying in $D_{n}$ and all points $P$ of $\gamma_{n}$ such that the tangent line to $\gamma_{n}$ at $P$ passes through $z=0$. Since $\gamma_{n}$ is an analytic curve, $E_{n}$ is finite. Let $\mathcal{L}=\overline{\mathbb{R}}_{0} \cup \mathcal{L}^{\prime}$, where $\mathcal{L}^{\prime}=\bigcup L$ and the union is taken over all closed rays $L$ on the $z$-sphere, which issue from the origin and pass through $z_{P}$ for some $P \in E_{n}$. Let $\widehat{\mathcal{L}}$ be the lift of $\mathcal{L}$ onto $\mathcal{R}$. Then the set $D_{n}^{\prime}=D \backslash \widehat{\mathcal{L}}$ contains a finite number of connected components having $P_{0}$ on the boundary. Let $T_{1}, \ldots, T_{N}$ be the set of all such connected components enumerated in such a way that $T_{k}$ lies over the sector $S_{k}=\left\{z: \theta_{k-1}<\theta<\theta_{k}\right\}$, where $0=\theta_{0}<\theta_{2}<\cdots<\theta_{N}=2 \pi p$.

Then every $T_{k}$ can be considered as a planar triangle $T_{k}=T_{k}\left(v_{0, k}, v_{1, k}, v_{2, k}\right)$ in $S_{k}$ having its vertex $v_{0, k}$ at $z=0$ and the opposite side $e_{0, k}$ joining the boundary rays of $S_{k}$. In addition, $T_{k}$ is starlike with respect to $v_{0, k}$. Let $2 \pi p \alpha_{k}=\theta_{k}-\theta_{k-1}$ be the angle of $T_{k}$ at the vertex $v_{0, k}$. Then $0<\alpha_{k} \leq 1$ and $\sum_{k=0}^{N} \alpha_{k}=1$. Therefore by Lemma 1(1), we have

$$
m_{\zeta}\left(D_{n}, P_{0}\right) \leq \sum_{k=1}^{N} \alpha_{k}^{2} m_{\zeta}\left(T_{k} ; v_{0, k} \mid v_{1, k}, v_{2, k}\right)
$$

Using (2.4) and applying Lemma 2, we obtain

$$
m_{\zeta}\left(T_{k} ; v_{0, k} \mid v_{1, k}, v_{2, k}\right)=m_{z}\left(T_{k} ; v_{0, k} \mid v_{1, k}, v_{2, k}\right) \leq \alpha_{k}^{-2} \int_{\theta_{k-1}}^{\theta_{k}} \log d_{P_{0}}\left(t, T_{k}\right) d t
$$

for all $1 \leq k \leq N$. This together with (2.14) implies that

$$
m_{\zeta}\left(D_{n}, P_{0}\right) \leq \frac{1}{2 \pi p} \int_{0}^{2 \pi p} \log d_{P_{0}}\left(t, D_{n}\right) d t
$$


By Levi's theorem, $\int_{0}^{2 \pi p} \log d_{P_{0}}\left(t, D_{n}\right) d t \rightarrow \int_{0}^{2 \pi p} \log d_{P_{0}}(t, D) d t$ as $n \rightarrow \infty$. Using (2.3), we obtain that $m_{\zeta}\left(D_{n}, P_{0}\right)=(1 / 2 \pi) \log \left(r_{n}\left|g^{\prime}(0)\right|\right) \rightarrow m_{\zeta}\left(D, P_{0}\right)$ as $n \rightarrow \infty$. Therefore, (2.12) follows from (2.16). The proof of inequality (2.13) follows the same lines as above and therefore is omitted.

(b) To prove the equality assertion for (2.12), we slit $D$ along a radial segment $I(\varphi) \subset D$ joining $P_{0}$ and $\partial D$ in the direction defined by the vector $\vec{v}(\varphi)$. We assume here that $I(\varphi)$ does not contain branch points of $\mathcal{R}$. Then $I(\varphi)$ exists for a.e. $\varphi, 0 \leq \varphi \leq 2 \pi p$. The slit domain $D \backslash I(\varphi)$ may be considered as a triangle $T=T\left(v_{0}, v_{1}, v_{2}\right)$ on $\mathcal{R}$ with the vertex $v_{0}$ at $P_{0}$, whose sides $e_{1}$ and $e_{2}$ are the two sides of the segment $I(\varphi)$. Applying (2.5) and (2.13), we obtain

$$
m_{\zeta}\left(D, P_{0}\right) \leq m_{\zeta}\left(T ; v_{0} \mid v_{1}, v_{2}\right) \leq(1 / 2 \pi p) \int_{0}^{2 \pi p} \log d_{P_{0}}(t, D) d t .
$$

Equality holds in the first of these inequalities if and only if $f_{D}^{-1}$ maps $I(\varphi)$ onto a radius of $\mathbb{D}$. Since this holds for a.e. $0 \leq \varphi \leq 2 \pi p$, it follows that $\arg \left(f_{D}(z) / z\right)$ is constant for all $z \in \mathbb{D} \backslash\{0\}$. Since $P_{0}$ is a branch point of $\mathcal{R}$ of order $p \geq 0$, the latter implies that $f(z)=c z^{p}$ for some $c \neq 0$. Then, of course, $D$ is a $p$-sheeted disk centered at $P_{0}$. The proof of the equality statement for (2.13) is similar.

\section{Proof of Theorem 1}

Let $f$ satisfy the assumptions of the theorem and let $\zeta=w^{1 / p}$ be a uniformizing parameter near $P_{0}$. By (2.3), $\log \left|a_{p}\right|=2 \pi m_{\zeta}\left(f(\mathbb{D}), P_{0}\right)$, which when combined with inequality (2.12) implies (1.4). Using the equality statement of Lemma 3(1), we conclude that equality occurs in (1.4) if and only if $f(\mathbb{D})$ is a $p$-sheeted disk having a single branch point at $P_{0}$. Then, of course, $f(z)=c z^{p}$ for some $c \in \mathbb{C} \backslash\{0\}$.

\section{The HYPERBolic Metric AND POlARIZATION}

To prove Theorem 4, we will use a polarization comparison theorem for the hyperbolic metric $\left[9\right.$. Let $L$ be a directed straight line on $\mathbb{C}$ and let $H^{+}$and $H^{-}$ be the left half-plane and the right half-plane with respect to $L$, respectively. For $z \in \overline{\mathbb{C}}$, let $z^{*}$ denote the reflection of $z$ in $L$. The polarization $E_{L}$ of a given set $E \subset \overline{\mathbb{C}}$ with respect to $L$ is then defined by

$$
E_{L}=\left(\left(E \cup E^{*}\right) \cap \bar{H}^{+}\right) \cup\left(\left(E \cap E^{*}\right) \cap H^{-}\right),
$$

where $E^{*}=\left\{z: z^{*} \in E\right\}$.

Theorem 5 (9). Let $D$ be a domain on $\overline{\mathbb{C}}$ carrying the hyperbolic metric $\lambda_{D}(z)$. Let $D_{L}$ be the polarization of $D$ with respect to $L$. Then $D_{L}$ is an open (not necessarily connected) set on $\overline{\mathbb{C}}$ having a hyperbolic metric $\lambda_{D_{L}}$, and the following inequalities hold:

$$
\begin{aligned}
\lambda_{D_{L}}(z) \lambda_{D_{L}}\left(z^{*}\right) & \leq \lambda_{D}(z) \lambda_{D}\left(z^{*}\right), \\
\min \left\{\lambda_{D_{L}}(z), \lambda_{D_{L}}\left(z^{*}\right)\right\} & \leq \min \left\{\lambda_{D}(z), \lambda_{D}\left(z^{*}\right)\right\} .
\end{aligned}
$$

Further, equality holds in (4.2) for some $z \in D$ such that $z^{*} \in D$ or in (4.3) for some $z \in D$ if and only if $D_{L}$ coincides with $D$ up to symmetry with respect to $L$.

Theorem 5 implies earlier comparison and symmetrization results on the hyperbolic metric due to V. Jørgensen, D. Minda, and A. Weitsman; see a recent survey 11. Combining Theorem 5 with appropriate conformal mappings, we easily obtain 
modifications of inequalities (4.2) and (4.3) for polarizations with respect to circles, ellipses, and some other curves.

\section{Proof of Theorem 4}

Let $\mathcal{F}$ be the set of all functions $f$ analytic in $\mathbb{D}$ satisfying the assumptions of the theorem. Then $\mathcal{F}$ is a normal family. Indeed, if $f \in \mathcal{F}$, then $f$ omits points $w_{0}=\infty$, $w_{1}=r_{1}+i$, and $w_{2}=r_{2}-i$ for some $0 \leq r_{1}, r_{2} \leq 2$. Let $\mathcal{G}=\left\{g=\varphi_{f} \circ f: f \in \mathcal{F}\right\}$, where $\varphi_{f}(w)=\left(w_{1}-w_{2}\right)^{-1}\left(2 w-\left(w_{1}+w_{2}\right)\right)$. Then every $g \in \mathcal{G}$ omits in $\mathbb{D}$ three points $w=\infty, w=1$, and $w=-1$. Hence, $\mathcal{G}$ is normal by Montel's theorem. Then it is clear that the set $\mathcal{F}=\left\{\varphi_{f}^{-1} \circ g\right\}$ is also normal.

(a) To prove (1.9)(a), we consider the following maximization problem: Find

$$
\sup \left|f^{\prime}(0)\right| \quad \text { over all } f \in \mathcal{F} \text {. }
$$

Since $\mathcal{F}$ is normal and $f(0)=1$ for all $f \in \mathcal{F}$, it follows that the supremum in (5.1) is finite and there is an $F \in \mathcal{F}$ such that $\left|F^{\prime}(0)\right|=\sup _{f \in \mathcal{F}}\left|f^{\prime}(0)\right|$. We claim that $F=f_{\alpha}$ or $F=2-f_{\alpha}$ for some $\alpha \in \mathbb{R}$.

Let $\Omega=F(\mathbb{D})$. Since $F$ is extremal for (5.1), Littlewood's principle of subordination (see [3, Theorem 2.21]) implies that $F$ is a universal covering of $\Omega$ by $\mathbb{D}$. Hence, $\left|F^{\prime}(0)\right|=\lambda_{\Omega}^{-1}(1)$. Let $M=\{w: 0 \leq \Re w \leq 2\} \backslash F(\mathbb{D}), \alpha=\inf \{\Re w: w \in M\}$, and $\beta=\sup \{\Re w: w \in M\}$. If $\alpha=\beta$, then $\alpha \neq 1$ and $\Omega$ must be the half-plane $\{w: \Re w>\alpha\}$ if $\alpha<1$ or the half-plane $\{w: \Re w<\alpha\}$ if $\alpha>1$. In this case, the theorem easily follows.

Assume that $\alpha<\beta$. Let $u_{0}=1$ if $\alpha<1<\beta$ and $u_{0}=(\alpha+\beta) / 2$ otherwise. Let $L\left(u_{0}\right)$ be the vertical line through $w=u_{0}$ directed upward if $0<u_{0} \leq 1$ and downward if $1<u_{0}<2$. Let $\Omega_{1}$ be the polarization of $\Omega$ with respect to $L\left(u_{0}\right)$ and let $F_{1}$ be the universal covering of $\Omega_{1}$ by $\mathbb{D}$ such that $F_{1}(0)=1$. From (4.1), it is clear that $F_{1} \in \mathcal{F}$. Using (1.6) and (4.3), we obtain that $\left|F_{1}^{\prime}(0)\right|=$ $\lambda_{\Omega_{1}}^{-1}(1) \geq \lambda_{\Omega}^{-1}(1)=\left|F^{\prime}(0)\right|$. Hence, $F_{1}$ is also an extremal for (5.1) and we must have $\lambda_{\Omega_{1}}(1)=\lambda_{\Omega}(1)$. Then $\Omega_{1}$ coincides with $\Omega$ up to symmetry with respect to $L\left(u_{0}\right)$ by the equality statement of Theorem 5 .

Let $\Omega_{2}=\Omega_{1} \cup\left\{w: \Re w>u_{0}\right\}$ if $u_{0} \leq 1$ and $\Omega_{2}=\Omega_{1} \cup\left\{w: \Re w<u_{0}\right\}$ if $u_{0}>1$. Then, of course, $\Omega_{2} \supset \Omega_{1}$, and it follows from our assumptions that $\Omega_{2} \neq \Omega_{1}$. In particular, $\lambda_{\Omega_{2}}(1)<\lambda_{\Omega_{1}}(1)$.

Since $\Omega$ coincides with its polarization $\Omega_{1}$ up to symmetry with respect to $L\left(u_{0}\right)$, it follows from the definition of polarization that $\left\{w=t+i v: \alpha \leq t \leq u_{0}\right\} \backslash \Omega_{2} \neq \emptyset$ for every $v$ if $u_{0} \leq 1$ and $\left\{w=t+i v: u_{0} \leq t \leq \beta\right\} \backslash \Omega_{2} \neq \emptyset$ for every $v$ if $u_{0}>1$. This implies that $F_{2} \in \mathcal{F}$, where $F_{2}$ is the universal covering of $\Omega_{2}$ by $\mathbb{D}$ such that $F_{2}(0)=1$. Since $F_{1}$ is an extremal for (5.1), we have $\lambda_{\Omega_{2}}^{-1}(1)=\left|F_{2}^{\prime}(0)\right| \leq\left|F_{1}^{\prime}(0)\right|=$ $\lambda_{\Omega_{1}}^{-1}(1)$ contradicting the inequality $\lambda_{\Omega_{2}}(1)<\lambda_{\Omega_{1}}(1)$. This completes the proof of (1.9)(a) together with the cases of equality.

(b) For $0<r<1$, let $M_{f}(r)=\max _{0 \leq \theta \leq 2 \pi}\left|f\left(r e^{i \theta}\right)-1\right|$. To prove (1.9)(b), we consider, for any given $0<r<1$, the problem of finding maximum

$$
M(r):=\max M_{f}(r) \quad \text { over all } f \in \mathcal{F} .
$$

Since $\mathcal{F}$ is normal there is an $F \in \mathcal{F}$ extremal for $(5.2)$. Let $\Omega=F(\mathbb{D})$ and let $\alpha$, $\beta, \Omega_{1}, \Omega_{2}$, and $F_{1}, F_{2}$ be the bounds, domains, and functions defined as in part (a) for our new $F$. If $\alpha=\beta$, then the theorem easily follows. 
Assume that $\alpha<\beta$. It follows from Theorem 14 in 9 that $M_{F_{1}}(r)>M_{F}(r)$ unless $\Omega_{1}$ coincides with $\Omega$ up to symmetry with respect to $L\left(u_{0}\right)$. Hence, $F_{1}$ is also an extremal for the problem (5.2). Then using the subordination principle (see [4, Theorem 2.21]), we conclude that $M_{F_{2}}(r)>M_{F_{1}}(r)$ unless $\Omega_{2}=\Omega_{1}$. Since $\Omega_{2} \neq \Omega_{1}$ in the case under consideration, the latter contradicts the extremality of $F_{1}$ for (5.2). Now the desired conclusion follows.

\section{Remarks}

The method discussed in Section 2 was developed in [8, [10]. A variant of this technique was used in the preprint [7] to prove the following analog of (1.4) for the inner radius:

$$
\log r\left(D, z_{0}\right) \leq(1 / 2 \pi) \int_{0}^{2 \pi} \log d_{z_{0}}(t, D) d t
$$

We remind the reader that the inner radius $r\left(D, z_{0}\right)$ of a domain $D$ having Green's function $g\left(z, z_{0}\right)$ with a pole at $z_{0} \in D$ is defined by

$$
\log r\left(D, z_{0}\right)=\lim _{z \rightarrow z_{0}}\left(g\left(z, z_{0}\right)+\log \left|z-z_{0}\right|\right) .
$$

In the case when $D$ is simply connected and starlike with respect to $z_{0}$, inequality (6.1) is due to G. Pólya and G. Szegö [6, p. 225].

The standard way to extend the Schwarz lemma for functions with a multiple point at $z=0$ is to factor $f(z)-f(0)$ as $z^{p} g(z)$ and then apply the basic case of the lemma to $g(z)$. We note that this approach does not work in the context of Theorem 1 since the factor $g(z)$ does not satisfy the requirements of this theorem in general.

\section{REFERENCES}

1. A. F. Beardon, D. Minda, The hyperbolic metric and geometric function theory. Quasiconformal Mappings and Their Applications, pp. 10-56. Editors: S. Ponnusamy et al., Narosa Publishing House, New Dehi, India, 2007.

2. R. B. Burckel, D. E. Marshall, D. Minda, P. Poggi-Corradini and T. J. Ransford, Area capacity and diameter versions of Schwarz's lemma. arXiv:0801.3629v1 [math.CV], 23 Jan 2008.

3. W. K. Hayman, Subharmonic Functions. Vol. 1. London Mathematical Society Monographs, No. 9, Academic Press, Inc., London, 1976. MR0460672 (57 665)

4. W. K. Hayman, Subharmonic Functions. Vol. 2. London Mathematical Society Monographs, No. 20, Academic Press, Inc., London, 1989. MR1049148 (91f:31001)

5. J. A. Jenkins, Univalent Functions and Conformal Mapping. Second edition, Springer-Verlag, New York, 1965. MR0096806 (20:3288)

6. G. Pólya and G. Szegö, Isoperimetric Inequalities in Mathematical Physics. Ann. Math. Studies 27, Princeton Univ. Press, 1952. MR0043486 (13:270d)

7. A. Yu. Solynin, Some estimates for the capacity of a condenser and the inner radius of a domain (in Russian). Preprint of Kuban State University, Krasnodar, 1983. Deponirovano in VINITI, no. 2015, 18 pp.

8. A. Yu. Solynin, Solution of a Pólya-Szegö isoperimetric problem. Zap. Nauchn. Semin. LOMI 168 (1988), 140-153; English translation in J. Soviet Math. 53 (1991), 311-320. MR0982489 (90h:30059) 
9. A. Yu. Solynin, Functional inequalities via polarization. Algebra i Analiz 8 no. 6 (1996), 148185; English translation in St. Petersburg Math. J. 8, no. 6 (1997), 1015-1038. MR.1458141 (98e:30001a)

10. A. Yu. Solynin, Moduli and extremal metric problems. Algebra i Analiz 11, no. 1 (1999), 3-86; English translation in St. Petersburg Math. J. 11, no. 1 (2000), 1-65. MR.1691080 (2001b:30058)

Department of Mathematics and Statistics, Texas Tech University, Box 41042, LubBock, Texas 79409

E-mail address: alex.solynin@ttu.edu 